\title{
Does Output Gap, Labour's Share or Unemployment Rate
}

\section{Drive Inflation?}

\section{Lanne, Markku}

2014

Lanne , M \& Luoto , J 2014 , ' Does Output Gap, Labour's Share or Unemployment Rate

Drive Inflation? ' , Oxford Bulletin of Economics and Statistics , vol. 76 , no. 5 , pp. 715-726 . https://doi.org/10.1111/c

http://hdl.handle.net/10138/223797

https://doi.org/10.1111/obes.12041

acceptedVersion

Downloaded from Helda, University of Helsinki institutional repository.

This is an electronic reprint of the original article.

This reprint may differ from the original in pagination and typographic detail.

Please cite the original version. 
This is the peer reviewed version of the following article: Lanne, M., and J. Luoto (2014), Does Output Gap, Labour's Share or Unemployment Rate Drive Inflation? Oxford Bulletin of Economics and Statistics, Volume 76, Issue 5, Pages 715 - 726, which has been published in final form at DOI: 10.1111/obes.12041. This article may be used for non-commercial purposes in accordance with Wiley Terms and Conditions for Self-Archiving.

\title{
Does Output Gap, Labor's Share or Unemployment Rate Drive Inflation?*
}

\section{MARKKU LANNE $\dagger$ and JANI LUOTO $\ddagger$}

$\dagger$ Department of Political and Economic studies, University of Helsinki, Finland, email: markku.lanne@helsinki.fi $\ddagger$ Department of Political and Economic studies, University of Helsinki, P.O.Box 17 (Arkadiankatu 7),FIN-00014 University of Helsinki, Finland, e-mail: jani.luoto@helsinki.fi

\begin{abstract}
We propose a new methodology for ranking in probability the commonly proposed drivers of inflation in the New Keynesian model. The approach is based on Bayesian model selection among restricted VAR models, each of which embodies only one or none of the candidate variables as the driver. Simulation experiments suggest that our procedure is superior to the previously used conventional pairwise Granger causality tests in detecting the true driver. Empirical results lend little support to labor share, output gap or unemployment rate as the driver of U.S. inflation.
\end{abstract}

JEL Classification C11, C52, C32, E31

Keywords: Inflation, new Keynesian Phillips curve, Bayesian variable selection

Word count: 5337

\footnotetext{
* We are grateful to Henri Nyberg and Antti Ripatti for useful comments. Financial support from the Academy of Finland is gratefully acknowledged.
} 


\section{Introduction}

According to the basic New Keynesian model, inflation is driven by expected future marginal costs, and this is embodied in the new Keynesian Phillips curve (NKPC). Unfortunately, taking the NKPC to data is hampered by the fact that marginal costs are not directly observable, and therefore, a number of theoretically well-motivated proxies have been proposed in the previous literature, including the labor's share of total income, various measures of the output gap, and more traditionally, the unemployment rate. However, different proxies tend to produce different estimates of the parameters of the NKPC and even yield different conclusions concerning its validity.

There are a large number of studies that find evidence in favor of each of the commonly proposed drivers of inflation (see, e.g., Galí and Gertler (1999), and Rudd and Whelan (2005)). In contrast to the previous literature, in this paper, we compare the three commonly proposed drivers of inflation mentioned above in a unified framework which also allows for the possibility that none of the suggested candidate variables is the true driver. In our analysis, the starting point is the implication of the new Keynesian model that in order for a certain variable to drive inflation, lagged inflation must be its useful predictor, provided inflation dynamics are well described by an autoregression (see Section 2 below). In other words, there should be Granger causality from inflation to its driver. While such Granger causality tests have previously been conducted, they have been isolated, considering only one variable at a time, and, thus the possibility that the true driver is not included in the set of the candidate variables considered has not been explicitly entertained.

In our analysis, we rank a number of restricted vector autoregressive (VAR) models by their Bayesian posterior probabilities. The models comprise inflation and the candidate driving variables, and each of them embodies Granger causality from inflation to only one of the candidates or none of them. In addition to the benefit of explicitly including the alternative that none of the candidates is the true driver, our procedure 
has statistical advantages. In particular, our Monte Carlo simulation experiments indicate superiority of the proposed procedure compared to the conventional Granger causality test that seems unable to discriminate against proxies correlated with the true driver.

With U.S. data since 1955, we find it very unlikely that any of our three candidates (real labor cost, output gap and unemployment rate) is driving inflation. The conventional Granger causality test, in contrast, lends support to both the output gap and unemployment rate as the driver. Our findings need not be interpreted as evidence against the New Keynesian model, but it may be that the true driver of inflation is not satisfactorily measured by the candidate variables considered in the previous literature, and hence care must be taken when interpreting results based on these proxies.

The plan of the rest of the paper is as follows. In Section 2, we discuss the new Keynesian Phillips curve and briefly survey a number of previous empirical results. Our econometric methodology is described in Section 3, while simulation results illustrating its properties and making comparisons to the conventional Granger causality test are presented in Section 4. Empirical results on U.S. data are discussed in Section 5. Finally, Section 6 concludes.

\section{New Keynesian Phillips Curve}

As already pointed out in the Introduction, according to the basic New Keynesian model, inflation $\pi_{t}$ is driven by expected future marginal costs $m c_{t}$, i.e.,

$$
\pi_{t}=\lambda \sum_{k=0}^{\infty} \beta^{k} E_{t}\left(m c_{t+k}\right),
$$

where $\beta$ is the subjective discount factor and $\lambda$ depends on the frequency of price adjustment and $\beta$. This result arises from the assumption due to Calvo (1983) that in every period, each identical firm has a fixed probability of adjusting the price of the differentiated product that it produces. Equation (1) can equivalently be written 


$$
\pi_{t}=\lambda m c_{t}+\beta E_{t}\left(\pi_{t+1}\right)
$$

which is often referred to as the New Keynesian Phillips curve (NKPC). As shown by Galí and Gertler (1999), there is an approximate log-linear relationship between the marginal cost and the output gap $x_{t}$, defined as the deviation of (the logarithm of) aggregate output from its equilibrium level in the absence of nominal rigidities, such that $m c_{t}=\kappa x_{t}$ with $\kappa$ the output elasticity of marginal cost. Hence, model (2) can alternatively be written as

$$
\pi_{t}=\lambda \kappa x_{t}+\beta E_{t}\left(\pi_{t+1}\right) .
$$

While equation (3) has been prominent in earlier empirical work on the Phillips curve, empirical research concerning the NKPC has been based on both (2) and (3), and there is an ongoing debate on which of these variables actually drives inflation (if either) and how they should be measured. Since neither variable is directly observable, a number of proxies have been entertained. Recently, Nason and Smith (2008) compared the properties of the U.S. output gap estimated by seven different methods and found little difference between them as drivers of inflation. In our empirical analysis, following the previous literature, we compute the output gap by applying the Hodrick-Prescott filter to the logarithmic real GDP per capita. As to equation (2), Galí and Gertler (1999) advocated measuring the real marginal cost by labor's share of income. This measure has subsequently been used in a number of empirical studies of the NKPC, and it has, to some extent, turned out more successful in producing results in accordance with prior expectations. However, Rudd and Whelan (2005), inter alia, have forcefully criticized against its use in estimating the NKPC. Another proxy for the marginal cost used in the empirical literature is the unemployment rate.

Instead of fixing a certain proxy variable, Basistha and Nelson (2007), Lanne and Luoto (2011), and Matthes and Wang (2012) have attempted to estimate the driver of inflation in the New Keynesian model as a latent variable that fits the NKPC or its hybrid version including also lagged inflation (Galí and Gertler, 1999) by construction. 
The time series of the output gap produced by Basistha and Nelson's (2007) model exhibits rather strong correlation with some of the output gap proxies entertained in the previous literature. Matthes and Wang (2012), on the other hand, find their latent driver of inflation the most strongly correlated with the labor share, while the correlations with various measures of the output gap are negligible. However, such inference on the strength of correlation between the latent and observed time series is potentially problematic for a number of reasons. First, it does not take into account the estimation error involved in computing the latent variable. Second, the process of the latent variable is dependent on the details of the assumed model that need not be correct. Finally, it is difficult to judge how large correlation is sufficient to deem one candidate variable as the true driver.

In gauging the validity of the different proxies as drivers of inflation, our starting point is the implication of the NKPC that in order for a certain variable $y_{t}$ to drive inflation, it must be predictable by lagged inflation. That this is the case can be seen by noting the well-known fact that (U.S.) inflation dynamics are well captured by the reduced-form equation

$$
\pi_{t}=A(L) \pi_{t-1}+\alpha y_{t}
$$

where $A(L)$ is a polynomial in the usual lag operator $L$. If the NKPC is the correct structural description of inflation dynamics, it must be the case that the significance of lags of inflation in (4) stems purely from its serving as a proxy for expected future values of $y_{t}$ in an equation of the form (1). Augmenting the NKPC by lagged inflation does not overturn this, i.e., inflation must have predictive power for future values of its driver even in the case of the hybrid NKPC. Rudd and Whelan (2005), inter alia, have tested this implication for the labor share by running tests of Granger causality from inflation, finding little predictive power. Our empirical results in Section 5 reconfirm this result for the labor share, but suggest that the output gap could potentially be a driver of U.S. inflation.

The aforementioned approach based on Granger causality tests is somewhat re- 
strictive in that it considers only one proxy variable at a time which makes it difficult to determine the overall size of the procedure in case none of the candidate variables is the true driver of inflation. Moreover, our simulation results in Section 4 indicate that when the proxy and true driver are correlated, the Granger causality test tends to overreject, failing to rule out a false driver. Therefore, we propose a new Bayesian procedure where the idea is to incorporate all potential drivers into the analysis simultaneously. Specifically, as will be discussed in detail in Section 3, the procedure involves estimating a number of restricted vector autoregressive (VAR) models containing all the candidate proxy variables, with inflation acting as a predictor of only one or none of them at a time. These models are subsequently compared by means of their posterior probabilities to find out whether any of the candidate variables is likely to drive inflation, and in case there are multiple drivers with positive probability, how they are ranked.

\section{$3 \quad$ Methodology}

The starting point of our econometric analysis is a $\operatorname{VAR}(\mathrm{p})$ model for inflation $\pi_{t}$ and the q candidate variables $x_{t}^{1}, \ldots, x_{t}^{q}$, and we estimate $q+1$ models, denoted by $M_{i}(i=$ $0,1, \ldots, q)$, obtained by placing different restrictions on it. In model $M_{i}$, the coefficients of the lags of inflation in the equations of all the other candidate variables but $x_{t}^{i}$ are set to zero, indicating that inflation Granger causes only $x_{t}^{i}{ }^{1}$ Repeating this for all the candidate variables $x_{t}^{i}, i=1,2, \ldots, q$, thus yields a set of models, each of which corresponds to one potential driver of inflation. Finally, model $M_{0}$ is obtained by restricting to zero the lags of inflation in the equations of all the candidate variables, in accordance with the idea that none of the proposed variables is the true driver.

\footnotetext{
${ }^{1}$ To be able to rank the restricted models, they must all be based on the same dependent variables. Thefore, all the candidate variables are included in the VAR model. Without further restrictions, these models are potentially overparametrized. Thus, to reduce the number of estimated parameters, we also set to zero the coefficients of the lags of the other candidate variables in all equations except in that of inflation.
} 
The comparison of the $q+1$ different models is based on their posterior model probabilities, which can be interpreted as probabilities of Granger causality from inflation to each of the candidate variables in turn (models $M_{1}, M_{2}, \ldots, M_{q}$ ) or to none of them (model $M_{0}$ ). If model $M_{0}$ turns out to have the greatest posterior probability, this can be interpreted in favor of none of the candidate variables being a plausible driver of inflation. Otherwise, model $M_{i}$ obtaining the greatest posterior probability is interpreted as $x_{t}^{i}$ being the likeliest driver. Of course, the more the greatest probability deviates from the rest, the stronger is the evidence in favor of the model in question. Compared to the conventional pairwise Granger-causality test, our approach has a number of benefits. First, because model $M_{0}$ is included, it provides a straightforward way of checking whether any of the candidate variables is the driver. Second, it yields a ranking of the different candidate variables in terms of posterior probabilities unlike separate Granger-causality tests that only allow for precluding some of the variables. Furthermore, the simulation experiments reported in Section 4, indicate that our procedure is superior in detecting the true driver of inflation among a set of candidate variables correlated with the true driver.

The posterior model probability of model $M_{i}$ is given by

$$
p\left(M_{i} \mid Y\right)=\frac{p\left(Y \mid M_{i}\right) p\left(M_{i}\right)}{\sum_{j=0}^{q} p\left(Y \mid M_{j}\right) p\left(M_{j}\right)},
$$

where $Y$ contains all the variables in the VAR model, $p\left(M_{i}\right)$ denotes the prior probability that $M_{i}$ is the true model (given that one of them is) ${ }^{2}$ and $p\left(Y \mid M_{i}\right)=$ $\int p\left(Y \mid \boldsymbol{\theta}_{i}, M_{i}\right) p\left(\boldsymbol{\theta}_{i} \mid M_{i}\right) d \boldsymbol{\theta}_{i}$ is the marginal likelihood with $p\left(\boldsymbol{\theta}_{i} \mid M_{i}\right)$ the prior density of all the parameters in $\boldsymbol{\theta}_{i}$ in model $M_{i}$, and $p\left(Y \mid \boldsymbol{\theta}_{i}, M_{i}\right)$ is the likelihood of $Y$ in

\footnotetext{
${ }^{2}$ Note that in case all the models are misspecified, i.e., when none of them is the true model, the weight (posterior probability) of the best model in the Kullback-Leiber metric tends to 1 asymptotically (see, for example, Fernández-Villaverde and Rubio-Ramírez, 2004). Hence, in particular, model $M_{0}$ obtaining the greatest posterior probability can be interpreted either as evidence against the New Keynesian model in general or as an indication that the true driver of inflation is not included in the set of candidate variables.
} 
model $M_{i}$.

To facilitate estimation of the restricted models, the unrestricted $\operatorname{VAR}(p)$ model is first written in the SUR form (see, e.g., Hamilton (1994, 315)). Model comparisons are based on approximate posterior model probabilities corresponding to the Schwarz Bayesian Information Criterion (BIC) (Schwarz, 1978). The logarithm of the approximate marginal likelihood of $M_{i}$ is defined as

$$
\ln p\left(M_{i} \mid Y\right) \approx c-\frac{1}{2} B I C_{i}
$$

where, for a model $i$ with $k$ free parameters, $B I C_{i}=-2 \times l+k \times \ln (T)$ with $l$ the value of the $\log$ likelihood function evaluated at the maximum likelihood (ML) estimate of $\boldsymbol{\theta}_{i}$, and $c$ is a constant. The approximation (6) can be obtained from the posterior density $p\left(\boldsymbol{\theta}_{i} \mid Y, M_{i}\right)$ by using the so-called Unit Informative Prior (UIP) for $\boldsymbol{\theta}_{i}$, which is multivariate normal with mean and covariance matrix equal to the ML estimate and $T$ times its asymptotic covariance matrix, respectively (see Raftery, 1995, and Sala-i-Martin et al., 2004). Perhaps the most popular parameter priors considered in the variable selection literature are Zellner's (1986) natural conjugate g-prior and some of its variants such as those proposed by Fernández et al. (2001), but we prefer to use the UIP because of its superior predictive performance (see, e.g., Eicher et al. (2011)). ${ }^{3}$ In addition to the parameter priors, we also need to specify the model prior probabilities $p\left(M_{i}\right)$. Given our empirical problem (in which the set of potential drivers of inflation is relatively small), it seems reasonable to assume that the models are equally likely a priori, that is, $p\left(M_{i}\right)=1 /(q+1)$ for each $i=0, \ldots, q$.

\section{Simulation Study}

We demonstrate the performance of the method put forth in Section 3 and compare it to the conventional Granger causality test by means of Monte Carlo simulation experiments. The data are generated from a typical three-equation dynamic stochastic

\footnotetext{
${ }^{3}$ Notice that the UIP prior depends on the data and thus violates the Bayes rule (see, e.g., Fernández et al., 2001), but this is the case for the g-priors here as well.
} 
general equilibrium (DSGE) model consisting of the (hybrid) NKPC generating inflation $\pi_{t}$, the dynamic IS curve for the output gap $x_{t}$, and the Taylor rule determining the interest rate $R_{t}$ :

$$
\begin{aligned}
\pi_{t} & =\gamma_{f} E_{t} \pi_{t+1}+\gamma_{b} \pi_{t-1}+\gamma x_{t}+\epsilon_{\pi t} \\
x_{t} & =\beta_{f} E_{t} x_{t+1}+\beta_{b} x_{t-1}-\beta_{r}\left(R_{t}-E_{t} \pi_{t+1}\right)+\epsilon_{x t} \\
R_{t} & =(1-\rho)\left(\omega_{\pi} \pi_{t}+\omega_{x} x_{t}\right)+\rho R_{t-1}+\epsilon_{R t}
\end{aligned}
$$

The errors are assumed to follow $\mathrm{AR}(1)$ processes:

$$
\begin{array}{rlrl}
\epsilon_{\pi t} & =\rho_{\pi} \epsilon_{\pi, t-1}+u_{\pi t}, & u_{\pi t} \sim N\left(0,0.5^{2}\right), \\
\epsilon_{x t} & =\rho_{x} \epsilon_{x, t-1}+u_{x t}, & u_{x t} \sim N\left(0,0.288^{2}\right), & \text { and } \\
\epsilon_{R t} & =\rho_{R} \epsilon_{R, t-1}+u_{R t}, & u_{R t} \sim N\left(0,0.252^{2}\right) .
\end{array}
$$

In the simulation experiments, we use the following benchmark parameter values. The variances of all the shocks as well as the $\operatorname{AR}(1)$ coefficients $\rho_{x}$ and $\rho_{R}$, which are assumed to take the values $\rho_{x}=0.5$ and $\rho_{R}=0.8$, respectively, are obtained from Lindé (2005). Following Galí and Gertler (1999), $\gamma$ in the NKPC is set at 0.05, while different combinations of the values of the parameters $\gamma_{f}$ and $\gamma_{b}$ are entertained with varying weights of inflation expectations and past inflation. However, to facilitate comparison, we fix the sum of these coefficients (at 0.96). Following Smets and Wouters (2007), among others, we assume that the price mark-up shock (cost push shock) $\epsilon_{\pi t}$ is (positively) serially correlated. We experimented with a number of different values of $\rho_{\pi}$, and found that the higher $\rho_{\pi}$ is, the better is the performance of our method. The reported results are based on $\rho_{\pi}=0.3$, which is relatively small compared to the empirical evidence of Smets and Wouters (2007). As far as the IS curve is concerned, we set $\beta_{f}=0.28, \beta_{b}=0.68$, and, following Nason and Smith (2008), $\beta_{r}=0.5 .^{4}$ Following Lindé (2005), the Taylor rule parameters are set at $\omega_{\pi}$ $=1.5, \omega_{x}=0.5$ and $\rho=0.5$, allowing for moderate interest rate smoothing.

\footnotetext{
${ }^{4}$ In addition to these benchmark parameter values of the IS curve, we also considered setting $\beta_{f}=0.68$ and $\beta_{b}=0.28$, indicating a larger role for forward-looking behavior. The conclusions
} 
In addition to the true driver of inflation $x_{t}$ (denoted $x_{t}^{1}$ henceforth), we generate two proxies with varying degrees of similarity with it. The first proxy is obtained as $x_{t}^{2}=x_{t}^{1}+\tau z_{t}$, where $z_{t}$ is an independent standard normal random variable. This formulation allows us to analyze how our method (and the Granger causality test) perform when the proxy and the true driver are correlated. We present the results based on $\tau=1$, yielding a relatively close proxy for $x^{1}$. Simulations based on $\tau=3$ yielding a less accurate proxy (not reported), produce qualitatively identical results. The other proxy that is completely unrelated to $x_{t}^{1}$ is generated by an $\operatorname{AR}(1)$ process $x_{t}^{3}=\phi x_{t-1}^{3}+\varepsilon_{t}$ for $t=1, \ldots, T$, where $\varepsilon_{t} \sim N(0,1)$. The reported results are based on $\phi=0.7$, but our experimentation with a number of different values of $\phi$ indicates the results hardly depend on the particular value of $\phi$.

For each combination of the parameter values, we generate 10000 realizations of $T=100$ and $T=200$ observations, roughly corresponding to the sample sizes to be considered in Section 5. To mitigate initialization effects, the first 200 observations at the beginning of each realization are discarded. For every realization, we calculate the posterior probabilities of Granger causality from inflation to each of the candidate variables in turn.

In the upper panel of Table 1, we report, for each model, the relative frequency of the posterior model probability exceeding $50 \%$. These figures indicate how often each model would result by the decision rule of selecting the model if its posterior probability is greater than $50 \%$. With relatively backward-looking inflation $\left(\gamma_{f}=\right.$ 0.28 and $\left.\gamma_{b}=0.68\right)$, the true model, i.e., the true driver of inflation, would always be selected, and even with more forward-looking inflation $\left(\gamma_{f}=0.68\right)$, it would be selected $96.6 \%$ and $99.9 \%$ of the time with 100 and 200 observations, respectively. The average posterior probabilities reported in the lower panel of the table tell a similar story. They are virtually indistinguishable from unity for the the true driver, with slightly smaller values with more forward-looking inflation and smaller sample remained virtually intact irrespective of the parameterization used, with our method performing slightly worse. The detailed results (not reported) are available upon request. 
size. Hence, it indeed seems that if the true driver is included in the set of candidate variables, our procedure is extremely efficient in finding it.

We also considered the case, where the true driver of inflation is not included among the candidate variables. Here the three candidate variables are two $\operatorname{AR}(1)$ processes generated as above with the autoregressive parameter $\phi$ taking values 0.4 and 0.7, and an independent standard normal random variable. Irrespective of specific parameter values or sample size, our procedure coupled with the $50 \%$ posterior probability rule selects the correct model where none of the candidates is included, approximately $90 \%$ of the time.

For comparison, we checked the performance of the standard Granger causality test discussed in Section 3. In other words, for each realization, we computed the $\mathrm{p}$ value of the test of Granger causality from inflation to each of the candidate variables in turn in bivariate $\operatorname{VAR}(1)$ models including inflation and the candidate variable in question. The rejection rates in Table 2 indicate that, irrespective of the sample size or of how forward-looking inflation is, the test has very high power against the hypothesis of no Granger causality from inflation to the true driver $\left(x_{t}^{1}\right)$ as well as the proxy correlated with the true driver $\left(x_{t}^{2}\right)$. Conversely, for the other proxy $\left(x_{t}^{3}\right)$ uncorrelated with true driver, the power of test is close to its size. Thus, the standard Granger causality test seems reliable in indicating the true driver, but it does not necessarily help in discriminating against proxies correlated with it.

\section{Results}

In this section, we present the empirical results on U.S. inflation. All estimations are based on the seasonally adjusted quarterly U.S. inflation series based on the GDP implicit price deflator series for the period from 1955:1 to 20011:2. In addition to the entire sample period, results are reported for two subsample periods of equal length, 1955:1-1983:1 and 1983:2-2011:2, to gauge robustness. The latter period is characterized by the decline in macroeconomic volatility and the ensuing relatively 
tranquil era, often referred to as the Great Moderation. We consider two commonly used candidate drivers of the U.S. inflation discussed in Section 2 above, namely the output gap and the real unit labor cost, as well as a more traditional explanatory variable for inflation, the unemployment rate (specifically, the seasonally adjusted total civilian unemployment rate (16 years of age and older $)$ ). In line with the previous literature, the output gap is proxied by the logarithm of the real GDP per capita detrended using the Hodrick-Prescott filter. ${ }^{5}$ Following Galí and Gertler (1999), inter alia, we use the real unit labor cost measured as the logarithm of the nominal unit labor cost in the nonfarm business sector divided by the implicit GDP deflator as a proxy for real marginal costs. The source of all data is the FRED database of the Federal Reserve Bank of St. Louis.

As outlined in Section 3, we estimate four restricted VAR models and compute their posterior probabilities. In all models, four lags are required to make the residuals white noise (according to their autocorrelation and partial autocorrelation functions) ${ }^{6}$ The posterior model probabilities $p\left(M_{i} \mid Y\right)$ are presented in Table 3. As already discussed in Section 3, these can be interpreted as the probabilities of Granger causality from inflation to each of the candidate variables in turn (models $M_{1}$ (output gap), $M_{2}$ (real unit labor cost), and $M_{3}$ (unemployment rate)) or to none of them (model $\left.M_{0}\right)$. In all sample periods considered, $M_{0}$ is by far the likeliest model with the other models taking only negligible probabilities. Hence, there seems to be quite convincing evidence in favor of none of the candidate variables commonly entertained in the previous literature driving U.S. inflation. This need not be evidence against the New Keynesian model, but it may be that the true driver of inflation is not satisfactorily measured by the candidate variables considered.

\footnotetext{
${ }^{5}$ We set the smoothing parameter of the Hodrick-Prescott filter at 1600 . We also experimented with measures of the output gap based on linear and quadratic trends. Furthermore, the logarithm of the real GDP (instead of the GDP per capita) was considered. The conclusions remained intact irrespective of the series used. The detailed results (not reported) are available upon request.

${ }^{6}$ As a robustness check, we also calculated posterior model probabilities using $\operatorname{VAR}(p)$ models with $p=2, \ldots, 5$. The conclusions remain intact irrespective of the $\operatorname{VAR}(p)$ model.
} 
For comparison, we also report the results of the standard test of pairwise Granger causality from inflation to each of the candidate variables in Table 4 . In accordance with Rudd and Whelan (2005), there is little sign of Granger causality from inflation to the real unit labor cost, indicating that it cannot be driving inflation. As far as the other two candidates are concerned, Granger causality from inflation is found at the $10 \%$ level (for the output gap even at the $5 \%$ level) in the entire data as well as in the first subsample period. However, in the latter subsample period, none of the candidate variables appears a plausible driver of inflation at reasonable significance levels. Thus, it is only in this period that the results in Tables 3 and 4 yield the same conclusion. Given our simulation results in Section 4, the standard Granger causality test is useful only in ruling out some of the candidate variables, but it may spuriously indicate false drivers. This shows up in the results in Table 4 in that they only suggest that the real labor cost cannot be driving inflation, but two plausible candidates are still remaining. It is only our procedure, simultaneously allowing for each candidate or none of them being the driver, that leads us to reject all candidates.

\section{Conclusion}

In this paper, we have proposed a new procedure for checking which one of a number of candidate variables is driving inflation in a new Keynesian model. Our approach is based on the simple idea that for a certain variable to drive inflation in that model, inflation must have predictive ability for it. We compute the posterior probabilities of a set of VAR models, each of which incorporates Granger causality from inflation to one candidate driver at a time, or, to none of them. Compared to the previous literature, the benefits are twofold. First, our unified framework explicitly allows for the possibility that none of the candidate variables is driving inflation. Second, our method yields a ranking of the different candidate variables in terms of posterior model probabilities. Furthermore, Monte Carlo simulation results suggest that our procedure is superior in detecting the true driver, especially when the candidate 
variables are correlated.

Our empirical results indicate that neither the labor share nor the output gap are likely drivers of U.S. postwar inflation. Also the more traditional candidate, unemployment rate, gets little support. As a matter of fact, with more than $98 \%$ probability, none of these variables most commonly used in empirical studies of the NKPC, is the true driver of U.S. inflation. These findings should not necessarily be interpreted as evidence against the new Keynesian model, but they cast doubt on many previous empirical results inasmuch as they rely on these proxies for the marginal cost.

\section{References}

Basistha, A., Nelson, C.R., 2007. New measures of the output gap based on the forward-looking new Keynesian Phillips curve. Journal of Monetary Economics $54,498-511$.

Calvo, G.A., 1983. Staggered prices in a utility maximizing framework. Journal of Monetary Economics 12, 383-398.

Doppelhofer, G. 2008. Model Averaging in The New Palgrave Dictionary in Economics, 2nd edition. L. Blume and S. Durlauf (eds.).

Eicher, T., Papageorgiou, C., Raftery, A., 2011. Default Priors and Predictive Performance in Bayesian Model Averaging, with Application to Growth Determinants. Journal of Applied Econometrics 26, 30-55.

Fernández, C., Ley, E., Steel, M.F.J., 2001. Benchmark Priors for the Bayesian Model Averaging. Journal of Econometrics 100, 381-427.

Fernández-Villaverde, J., Rubio-Ramírez, J.F., 2004. Comparing dynamic equilibrium models to data: A Bayesian approach. Journal of Econometrics 123, $153-187$. 
Galí, J., Gertler, M., 1999. Inflation dynamics: a structural econometric analysis Journal of Monetary Economics 44, 195-222.

George, E. I., McCulloch, R. E., 1993. Variable selection via Gibbs sampling. Journal of the American Statistical Association 88, 881-889.

Hamilton, J.D., 1994. Time Series Analysis. Princeton University Press, Princeton, NJ.

Kass, R.E., Wasserman, L., 1995. A Reference Bayesian Test for Nested Hypotheses and its Relationship to the Schwarz Criterion. Journal of the American Statistical Association 90, 928-934.

Lanne, M., Luoto, J., 2011. Autoregression-Based Estimation of the New Keynesian Phillips Curve. HECER Discussion Paper 321.

Lindé, J., 2005. Estimating New-Keynesian Phillips curves: a full information maximum likelihood approach. Journal of Monetary Economics 52, 1135-1149.

Matthes, C., Wang, M.-C., 2012. What drives inflation in New Keynesian models? Economics Letters 113, 338-342.

Nason, J.M., Smith, G.W., 2008. The New Keynesian Phillips curve: lessons from single-equation econometric estimation. Economic Quarterly 94, 361-395. Raftery, A.E., 1995. Bayesian Model Selection for Social Research. Sociological Methodology 25, 111-163.

Rudd, J., Whelan, K., 2005. Does labor's share drive inflation? Journal of Money, Credit, and Banking 37, 297-312.

Sala-i-Martin, X., Doppelhofer, G., Miller, R.I., 2004. Determinants of LongTerm Growth: A Bayesian Averaging of Classical Estimates (BACE) Approach. American Economic Review 94, 813-835. 
Schwarz, G., 1978. Estimating the Dimension of a Model. Annals of Statistics $6,461-464$.

Smets F, Wouters, R., 2007. Shocks and frictions in US business cycles: a Bayesian DSGE approach. American Economic Review 97, 586-606.

Zellner, A., 1986. On Assessing Prior Distributions and Bayesian Regression Analysis with g-prior Distributions, in Bayesian Inference and Decision Techniques: Essays in Honor of Bruno de Finetti. Goel, P.K. and A. Zellner, eds., North-Holland, Amsterdam. 
Table 1: Simulation results of the Bayesian model averaging procedure.

\begin{tabular}{|c|c|c|c|c|c|c|c|c|c|}
\hline \multirow[b]{2}{*}{$\gamma_{f}$} & \multirow[b]{2}{*}{$\gamma_{b}$} & \multicolumn{8}{|c|}{ Sample Size } \\
\hline & & \multicolumn{4}{|c|}{$T=100$} & \multicolumn{4}{|c|}{$T=200$} \\
\hline & & \multicolumn{8}{|c|}{ Posterior Model Probability $>50 \%$} \\
\hline & & $M_{1}$ & $M_{2}$ & $M_{3}$ & $M_{0}$ & $M_{1}$ & $M_{2}$ & $M_{3}$ & $M_{0}$ \\
\hline 0.28 & 0.68 & 1.000 & 0.000 & 0.000 & 0.000 & 1.000 & 0.000 & 0.000 & 0.000 \\
\hline 0.48 & 0.48 & 1.000 & 0.000 & 0.000 & 0.000 & 1.000 & 0.000 & 0.000 & 0.000 \\
\hline \multirow[t]{3}{*}{0.68} & 0.28 & 0.966 & 0.000 & 0.002 & 0.016 & 0.999 & 0.000 & 0.000 & 0.000 \\
\hline & & \multicolumn{8}{|c|}{ Average Posterior Model Probability } \\
\hline & & $M_{1}$ & $M_{2}$ & $M_{3}$ & $M_{0}$ & $M_{1}$ & $M_{2}$ & $M_{3}$ & $M_{0}$ \\
\hline 0.28 & 0.68 & 0.999 & 0.000 & 0.000 & 0.001 & 1.000 & 0.000 & 0.000 & 0.000 \\
\hline 0.48 & 0.48 & 0.999 & 0.000 & 0.000 & 0.001 & 1.000 & 0.000 & 0.000 & 0.000 \\
\hline 0.68 & 0.28 & 0.945 & 0.007 & 0.009 & 0.039 & 0.998 & 0.000 & 0.000 & 0.002 \\
\hline
\end{tabular}

The entries are relative frequencies of the posterior model probability exceeding $50 \%$, and average posterior model probabilities in the upper and lower panels, respectively. The DGP is as described in Section 4 apart from the values of the parameters $\gamma_{f}$ and $\gamma_{b}$ given in the first two columns. Models $M_{i},(i=1,2,3)$, are $\operatorname{VAR}(1)$ models for inflation and the three candidate variables with the lags of inflation excluded from all the equations except that of the $i$ th candidate, while model $M_{0}$ is a $\operatorname{VAR}(1)$ model with the lags of inflation excluded from the equations of all the cadidate variables. The number of replications is 10000.

Table 2: Simulation results of the Granger causality tests.

\begin{tabular}{|c|c|c|c|c|c|c|c|}
\hline \multirow{3}{*}{$\gamma_{f}$} & \multirow{3}{*}{$\gamma_{b}$} & \multicolumn{6}{|c|}{ Sample Size } \\
\hline & & \multicolumn{3}{|c|}{$T=100$} & \multicolumn{3}{|c|}{$T=200$} \\
\hline & & $x_{t}^{1}$ & $x_{t}^{2}$ & $x_{t}^{3}$ & $x_{t}^{1}$ & $x_{t}^{2}$ & $x_{t}^{3}$ \\
\hline 0.28 & 0.68 & 0.997 & 0.996 & 0.067 & 1.000 & 1.000 & 0.063 \\
\hline 0.48 & 0.48 & 0.992 & 0.982 & 0.066 & 1.000 & 1.000 & 0.061 \\
\hline 0.68 & 0.28 & 0.718 & 0.666 & 0.065 & 0.927 & 0.902 & 0.057 \\
\hline
\end{tabular}

The DGP is as described in Section 4 apart from the values of the parameters $\gamma_{f}$ and $\gamma_{b}$ given in the first two columns. The entries are rejection rates of the $5 \%$ level tests of Granger causality from inflation to each candidate variable $x_{t}^{i}$, with $x_{t}^{1}$ the true driver, $x_{t}^{2}$ a proxy correlated with it, and $x_{t}^{3}$ the unrelated proxy. 
Table 3: Posterior model probabilities.

\begin{tabular}{llccc}
\hline \hline & & \multicolumn{3}{c}{ Sample Period } \\
\cline { 3 - 5 } Model & Granger Causality to & $1955: 1-2011: 2$ & $1955: 1-1983: 1$ & $1983: 2-2011: 2$ \\
\hline$M_{1}$ & Output Gap & 0.009 & 0.008 & 0.000 \\
$M_{2}$ & Real Labor Cost & 0.000 & 0.001 & 0.000 \\
$M_{3}$ & Unemployment Rate & 0.007 & 0.042 & 0.001 \\
$M_{0}$ & None of the above & 0.984 & 0.948 & 0.999
\end{tabular}

The entries are posterior probabilities of $\operatorname{VAR}(4)$ models where the coefficients of the lags of inflation in the equations of the variables other than the one in the first column are restricted to zero.

Table 4: Granger causality tests.

\begin{tabular}{lccc}
\hline \hline & \multicolumn{3}{c}{ Sample Period } \\
\cline { 2 - 4 } Granger Causality to & $1955: 1-2011: 2$ & $1955: 1-1983: 1$ & $1983: 2-2011: 2$ \\
\hline Output Gap & 0.002 & 0.009 & 0.503 \\
Real Labor Cost & 0.682 & 0.722 & 0.909 \\
Unemployment Rate & 0.077 & 0.007 & 0.101
\end{tabular}

The entries are $\mathrm{p}$ values of the standard test of Granger causality from inflation to the variable in the first column. In each case, the test is based on a VAR(4) model. 